\title{
The role of symmetry in attraction to average faces
}

\author{
Benedict C. Jones And Lisa M. DeBruine \\ University of Aberdeen, Aberdeen, Scotland \\ AND \\ Anthony C. LitTle \\ University of Stirling, Stirling, Scotland
}

\begin{abstract}
Although many studies have demonstrated that average faces tend to be attractive, few studies have examined the extent to which symmetry contributes to the attractiveness of average faces. Such studies are potentially important, however, because average faces are highly symmetric and increasing the symmetry of face images increases their attractiveness. Here we demonstrate that increasing averageness of 2-D face shape independently of symmetry is sufficient to increase attractiveness, indicating that preferences for symmetry cannot solely explain the attractiveness of average faces. Additionally, we show that averageness preferences are significantly weaker when the effects of symmetry are controlled for using computer graphic methods than when the effects of symmetry are not controlled for, suggesting that symmetry contributes to the attractiveness of average faces. Importantly, this latter finding was not explained by the greater perceived similarity between versions of faces that varied in averageness, but not symmetry, than between versions of faces that varied in both averageness and symmetry.
\end{abstract}

Many studies of facial attractiveness have demonstrated that people prefer average faces to relatively distinctive ones (see Rhodes, 2006, for a review). For example, composites (or prototypes) manufactured by averaging the shape and color information from a sample of faces are typically judged more attractive than their constituent faces (Langlois \& Roggman, 1990; Little \& Hancock, 2002; Rhodes, Sumich, \& Byatt, 1999; Valentine, Darling, \& Donnelly, 2004). Furthermore, morphing faces toward a more average configuration also increases their attractiveness (see, e.g., Rhodes, Yoshikawa, et al., 2001; Valentine et al., 2004). Although some researchers have suggested that preferences for average faces might reflect preferences for attractive nonaverage traits that composite images possess but that are artifacts of the computer graphic methods used in their manufacture (e.g., unnaturally smooth skin texture, Alley \& Cunningham, 1991; Benson \& Perrett, 1991), increasing the averageness of faces while not altering their skin condition is sufficient to increase attractiveness (Little \& Hancock, 2002; O’Toole, Price, Vetter, Bartlett, \& Blanz, 1999; Penton-Voak \& Perrett, 2001; Rhodes \& Tremewan, 1996). Attraction to average faces may simply be a by-product of processing biases in the visual system (see, e.g., Enquist, Ghirlanda, Lundqvist, \& Wachtmeister, 2002; Halberstadt \& Rhodes, 2000; Winkielman, Halberstadt, Fazendeiro, \& Catty, 2006) or reflect adaptations that promote associations with healthy individuals (Rhodes, Zebrowitz, et al., 2001; Thornhill \& Gangestad,
1993). Of course, these explanations are not necessarily mutually exclusive: Processing biases in the visual system may be the proximate mechanism that underpins adaptive preferences for healthy individuals.

Although a recent meta-analysis of findings from 18 papers that tested for a positive relationship between facial attractiveness and averageness reported a large effect size (mean effect size $=0.52$; Rhodes, 2006), only three studies have investigated the role of symmetry in preferences for average faces (Rhodes et al., 1999; Rhodes, Yoshikawa, et al., 2001; Valentine et al., 2004). Such studies are potentially important, however, since average faces are highly symmetric (Alley \& Cunningham, 1991) and increasing symmetry in face images increases their attractiveness (Little \& Jones, 2003; Perrett et al., 1999; Rhodes et al., 1998; but see also Swaddle \& Cuthill, 1995). Thus, attraction to symmetry may play an important role in preferences for average faces (Alley \& Cunningham, 1991).

Using linear regression, Rhodes et al. (1999) demonstrated that attractiveness ratings of individual faces were independently and positively related to both averageness ratings of the faces and measured facial symmetry. Rhodes, Yoshikawa, et al. (2001) also found that increasing the averageness of face images while holding symmetry constant increased the attractiveness of the faces. More recently, Valentine et al. (2004) demonstrated that increasing the averageness of profile views of faces, which have no bilateral axis of symmetry, increased their attractive-

B. C. Jones, ben.jones@abdn.ac.uk 
ness. Collectively, these findings suggest that preferences for average faces are not solely due to symmetry preferences (Rhodes, 2006). However, none of these studies established whether or not symmetry does actually contribute to preferences for average faces.

Although Valentine et al. (2004) found that preferences for averageness were weaker for judgments of profile views of faces than for judgments of front views of faces, this apparent effect of view may be an artifact of reduced visibility of cues to averageness in the profile views (Valentine et al., 2004) or a consequence of differences in the gaze direction between views. Findings from both neuroimaging and behavioral studies suggest that preferences for facial cues of physical attractiveness are stronger when the depicted individuals appear to be interested in the viewer than when they appear relatively disinterested in the viewer (Jones, DeBruine, Little, Conway, \& Feinberg, 2006; Kampe, Frith, Dolan, \& Frith, 2001; O’Doherty et al., 2003). Furthermore, in Valentine et al.'s study, preferences for averageness may have been a by-product of the methods used to manipulate the averageness of faces, as these did not dissociate the effects of increasing the averageness of face shape from the effects of increasing other nonaverage facial characteristics that are also important for attractiveness (e.g., smoothness of skin texture; Little \& Hancock, 2002). Indeed, studies have demonstrated that even very subtle differences in apparent skin quality can have pronounced effects on facial attractiveness (e.g., Jones et al., 2004; Little \& Hancock, 2002).

In light of the above, we conducted two experiments in order to (1) establish whether or not increasing the prototypicality (i.e., averageness) of the 2-D shape of face images is sufficient to increase their attractiveness when facial symmetry, color, and texture are not manipulated, and (2) establish whether or not increasing the prototypicality of the 2-D shape of face images has a greater effect on attractiveness judgments when effects of symmetry are not controlled for than when symmetry is held constant. We undertook this latter comparison to test if symmetry contributes to preferences for average faces.

\section{EXPERIMENT 1}

Here we tested the extent to which participants preferred versions of female faces in which the averageness of 2-D shape, but not color and texture, had been warped toward an average configuration (i.e., anti-caricatured versions) to the original (i.e., unmanipulated) versions. Furthermore, we compared the strength of averageness preferences when the original and anti-caricatured versions of the face images were both perfectly symmetric (perfect symmetry condition) and when possible effects of symmetry were not controlled in this way (normal symmetry condition). If averageness is attractive even when cues of symmetry are not visible, as is the case in the perfect symmetry condition, then increasing averageness will increase attractiveness in both the perfect symmetry and normal symmetry conditions. Nevertheless, if symmetry contributes to preferences for average faces then preferences for averageness will be greater in the normal symmetry condition than in the per- fect symmetry condition. We also investigated whether an effect of symmetry on preferences for average faces can be explained by greater perceived similarity of original and anti-caricatured faces in the perfect symmetry condition than in the normal symmetry condition.

\section{Method}

Stimuli. Full color, front view face images of 30 young female adults (age: $M=18.56$ years, $S D=0.72$ ) with neutral expressions were taken with a digital camera under standardized lighting conditions and against the same background. These images were then aligned to a standard interpupillary distance.

These 30 face images were then used to manufacture a female prototype face, with the average color and shape information for the sample and representative texture. The methods used to manufacture this prototype were identical in detail to those used to manufacture composites in previous studies of face preferences (e.g., DeBruine, 2005; Perrett et al., 1998; Perrett et al., 2002), including perceptions of average faces (e.g., Little \& Hancock, 2002). Briefly, the same 189 facial locations were first marked on each face image. A computer algorithm then calculated the average XY coordinates for each point and also calculated the average 2-D configuration (Rowland \& Perrett, 1995; Tiddeman, Burt, \& Perret, 2001). Next, the average RGB values for each pixel were calculated and applied to the average shape (Rowland \& Perrett, 1995; Tiddeman et al., 2001).

We manufactured versions of each of the 30 original (i.e., uncaricatured) female face images to have a more average 2-D shape (i.e., we created anti-caricatured versions) by morphing the 2-D shape of each image toward that of the female prototype by $50 \%$ of the vector differences in 2-D shape (see Rowland \& Perrett, 1995 and Tiddeman et al., 2001 for technical details). This method does not alter color and texture cues (Little \& Hancock, 2002). These uncaricatured and anticaricatured versions were used as stimuli in the normal symmetry condition of the experiment and examples are shown in Figure 1.

To manufacture stimuli for the perfect symmetry condition, the processes described above were repeated, using versions of the 30 original female face images that had first been made perfectly symmetric. Following Rhodes, Yoshikawa, et al. (2001), Jones et al. (2004), and

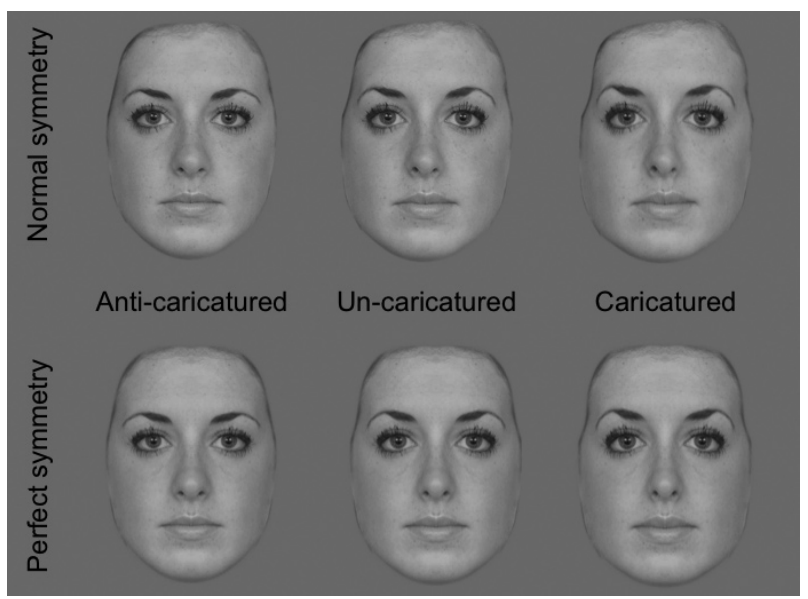

Figure 1. Examples of face images used in our experiments. In Experiment 1, participants were asked to indicate which face in each pair was more attractive when choosing between the anti-caricatured (leftmost column) and uncaricatured (middle column) versions with either normal (top row) or perfect (bottom row) symmetry. In Experiment 2, participants were asked to indicate which face in each pair was more attractive when choosing between the uncaricatured (middle column) and caricatured (rightmost column) versions with either normal (top row) or perfect (bottom row) symmetry. 
Cárdenas and Harris (2006), perfectly symmetric versions of each of the 30 original images were manufactured by averaging the shape, color, and texture information from each of the original images and their mirror-reversed counterparts. This was done separately for each of the 30 female identities. The resultant images (i.e., symmetric uncaricatured versions and symmetric anti-caricatured versions) were used as stimuli in the perfect symmetry condition of the experiment (see Figure 1 for examples). Note that this procedure ensures that both the uncaricatured and anti-caricatured versions of the perfectly symmetric faces did not differ in symmetry. Rhodes, Yoshikawa, et al. (2001) have previously used a similar method to manufacture face images that differ in averageness but do not differ in symmetry.

Thus, two sets of 30 pairs of images were manufactured in total: 30 pairs differing in averageness and symmetry, but matched in other regards (e.g., identity, skin color, and skin texture), that were shown in the normal symmetry condition, and 30 pairs differing in averageness of 2-D shape, but that were matched in identity, symmetry, skin color, and skin texture, that were shown in the perfect symmetry condition. All images shown in the experiment were masked so that hairstyle and clothing were not visible.

Procedure. Participants $(n=24,17$ female; ages: $M=23.72$ years, $S D=5.11$ ) viewed the 60 pairs of faces and were instructed to click on the face in each pair that they considered more attractive. Trial order and the side of the screen on which any particular image was presented were fully randomized.

A different group of 33 participants (age: $M=19.66$ years, $S D=$ 1.28; 29 female) viewed the 60 pairs of faces and were instructed to rate the similarity of the faces in each pair using a 1 (not very similar) to 7 (very similar) scale. Trial order and the side of the screen on which any particular image was presented were fully randomized. Participants were also instructed that the images shown would be different versions of the same individual (and would therefore look very similar) but that they should try to use the full range of the scale. This method for assessing the perceived similarity of face images is similar to those used in previous studies of face perception (e.g., Maloney \& Dal Martello, 2006).

Initial processing of data. For each of the 30 image identities (i.e., each item), the proportion of participants who chose the more average version (i.e., the anti-caricature) as the more attractive was calculated separately for the perfect symmetry and normal symmetry conditions. Additionally, the average similarity rating for each of the 30 image identities was calculated separately for the perfect symmetry and normal symmetry conditions. Interrater agreement for the similarity ratings (i.e., Cronbach's alpha) was .96.

\section{Results}

Two-tailed $p$ values are reported for all analyses. Onesample $t$ tests indicated that the proportion of participants choosing the anti-caricatured versions as the more attractive was significantly greater than chance (.5) in the normal symmetry condition $[t(29)=13.06, p<.001 ; M=0.81$, $S E=0.02]$ and in the perfect symmetry condition $[t(29)=$ 8.26, $p<.001 ; M=0.75, S E=0.03$ ]. A paired-samples $t$ test comparing these scores indicated that a greater proportion of participants chose the anti-caricatured versions in the normal symmetry condition than in the perfect symmetry condition $[t(29)=2.82, p=.009]$.

A paired-samples $t$ test comparing the perceived similarity of faces in the normal symmetry and perfect symmetry conditions indicated that pairs of faces in the perfect symmetry condition were perceived as being more similar than those shown in the normal symmetry condition $[t(29)=3.39, p=.002$; perfect symmetry condition: $M=5.19, S E=0.07$; normal symmetry condition: $M=$ 5.02, $S E=0.07]$.
Next, we used ANCOVA to test whether the difference between the perceived similarity of face-pairs in the normal symmetry and perfect symmetry conditions explains the stronger preference for averageness in the normal symmetry condition than in the perfect symmetry condition. The ANCOVA had one within-subjects factor (symmetry condition), with two levels (perfect, normal), and a covariate (the difference in perceived similarity of face-pairs in the perfect symmetry and normal symmetry conditions). The dependent variable was the proportion of participants who preferred the more average face. This analysis revealed a significant main effect of symmetry condition $[F(1,28)=5.51, p=.026]$, whereby a greater proportion of participants chose the anti-caricatured versions in the normal symmetry condition than in the perfect symmetry condition. There was no significant effect of the covariate $[F(1,28)=1.56, p=.22]$ and no significant interaction between the covariate and the within-subjects factor symmetry condition $[F(1,28)=0.00, p>.99]$.

\section{EXPERIMENT 2}

Findings from Experiment 1 suggest that increasing averageness increases the attractiveness of faces even when images do not differ in symmetry (i.e., preferences for averageness were observed in both the perfect symmetry and normal symmetry conditions). Our findings also suggest that symmetry contributes to preferences for average faces (i.e., preferences for averageness were greater in the normal symmetry condition than in the perfect symmetry condition). This effect remained when possible effects of the difference in the perceived similarity of face-pairs in the normal symmetry and perfect symmetry conditions were controlled using analysis of covariance.

In Experiment 1, we investigated preferences for average faces by testing participants' preferences for uncaricatured versions of female faces versus versions in which the averageness of 2-D shape had been increased (i.e., anti-caricatured versions). In Experiment 2, we tested whether the effects that we observed in Experiment 1 generalize to preferences for uncaricatured versions of female faces versus versions in which averageness of 2-D shape had been decreased (i.e., caricatured versions in which the distinctive aspects of the individual faces had been exaggerated).

\section{Method}

Stimuli. Using the same methods and face images that were used to manufacture stimuli for Experiment 1, versions of the 30 female faces were manufactured with decreased averageness of 2-D shape (i.e., caricatured versions). These caricatured versions were created by exaggerating the linear differences in 2-D shape between each image and the female prototype by $50 \%$ of the vector differences (i.e., by shifting the 2-D shape of the face images away from the prototypic shape). As in Experiment 1, manipulating facial averageness in this way does not alter skin color or texture.

Following Experiment 1, two sets of 30 pairs of images were manufactured in total: 30 pairs differing in both averageness and symmetry, but matched in other regards, such as identity, skin color, and skin texture (see Figure 1), that were used in the normal symmetry condition, and 30 pairs differing in averageness of 2-D shape, but matched 
in identity, symmetry, skin color and skin texture (see Figure 1), that were used in the perfect symmetry condition. As in Experiment 1, all images were masked so that hairstyle and clothing were not visible.

Procedure. The testing procedure was identical to that used in Experiment 1. Twenty-three individuals (12 female; ages: $M=$ 25.61 years, $S D=7.05$ ) made attractiveness judgments, and a different group of 42 individuals (26 female; ages: $M=22.32$ years, $S D=5.73$ ) rated the similarity of face-pairs. None of the participants in Experiment 2 had taken part in Experiment 1.

Initial processing of data. Initial processing of data was identical to that in Experiment 1, except that here the more average versions in each face-pair were the uncaricatured versions. Interrater agreement (i.e., Cronbach's alpha) for similarity ratings was .96

\section{Results}

Two-tailed $p$ values are reported for all analyses. Onesample $t$ tests indicated that the proportion of participants choosing the more average face in each pair as the more attractive face was significantly greater than chance (i.e., .5) in the normal symmetry condition $[t(29)=31.52$, $p<.001 ; M=0.89, S E=0.01]$ and in the perfect symmetry condition $[t(29)=21.93, p<.001 ; M=0.86, S E=$ $0.02]$. A paired-samples $t$ test indicated that a greater proportion of participants chose the more average faces in the normal symmetry condition than in the perfect symmetry condition $[t(29)=2.51, p=.018]$. A paired-samples $t$ test also indicated that pairs of faces in the perfect symmetry condition were judged more similar than those in the normal symmetry condition $[t(29)=4.98, p<.001$; perfect symmetry condition: $M=4.77, S E=0.07$; normal symmetry condition: $M=4.49, S E=0.07]$.

Following Experiment 1, we used ANCOVA to test whether the difference in the perceived similarity of facepairs in the perfect symmetry and normal symmetry conditions explained the stronger preference for averageness in the normal symmetry condition than in the perfect symmetry condition. The design of this ANCOVA was identical to the one reported in Experiment 1. The ANCOVA revealed a significant main effect of symmetry condition $[F(1,28)=5.09, p=.032]$, whereby a greater proportion of participants chose the more average faces in the normal symmetry condition than in the perfect symmetry condition. There was no significant effect of the difference in perceived similarity of face-pairs between conditions $[F(1,28)=2.21, p=.15]$ and no significant interaction between this covariate and the within-subjects factor symmetry condition $[F(1,28)=0.40, p=.53]$.

\section{DISCUSSION}

In each of our experiments, participants preferred the more average versions of the faces significantly more often than the less average (i.e., relatively distinctive) versions. These findings for preferences for average faces complement those from previous studies that have demonstrated the attractiveness of average faces (Langlois \& Roggman, 1990; Little \& Hancock, 2002; Rhodes et al., 1999; Rhodes, Yoshikawa, et al., 2001; Rhodes, Zebrowitz et al., 2001; Valentine et al., 2004). Furthermore, because skin color and texture were not manipulated in our stimuli, our findings for attraction to average faces are further evidence that the at- tractiveness of composite faces is not solely due to changes in skin smoothness when manipulating averageness (see also Little \& Hancock, 2002; O’Toole et al., 1999; PentonVoak \& Perrett, 2001; Rhodes \& Tremewan, 1996).

Significant preferences for the more average faces were also observed in each of our experiments when the effects of symmetry were controlled using computer graphic methods (i.e., when the average and relatively distinctive versions of the faces were both perfectly symmetric). This complements Rhodes et al.'s previous findings for linear regression analyses of facial symmetry, rated averageness and facial attractiveness, which showed that facial attractiveness was independently and positively related to both averageness and symmetry (Rhodes et al., 1999). While Valentine et al. (2004) showed that manipulating the averageness of profile faces, which have no bilateral axis of symmetry, influenced perceptions of their attractiveness, this finding is difficult to interpret because both averageness of 2-D shape and smoothness of skin texture were simultaneously manipulated in the stimuli. By contrast, here we show that increasing the averageness of 2-D shape while not altering symmetry, skin color, or skin texture is sufficient to increase the attractiveness of faces (see also Rhodes, Yoshikawa, et al., 2001).

Previous studies that have investigated the role of symmetry in preferences for average faces were not designed to be strong tests of the extent to which symmetry might contribute to the attractiveness of average faces, but were designed to test whether or not averageness was attractive independent of the effects of symmetry (Rhodes et al., 1999; Rhodes, Yoshikawa, et al., 2001; Valentine et al., 2004). By contrast, the design of our experiments allowed for strong tests of both of these issues. Although we show that preferences for average faces can occur independent of the effects of symmetry, our findings for greater preferences for average faces in the normal symmetry condition (i.e., when faces varied in both averageness and symmetry) than in the perfect symmetry condition (i.e., when faces varied in averageness but not in symmetry) suggest that symmetry does contribute to the attractiveness of average faces. While both of our experiments showed that facepairs were perceived to be less similar when they differed in averageness and symmetry (i.e., in the normal symmetry condition) than when they differed in averageness only (i.e., in the perfect symmetry condition), further analyses indicated that this difference did not fully account for stronger preferences for averageness in the normal symmetry condition than in the perfect symmetry condition. It is noteworthy that the contribution of symmetry to the attractiveness of average faces appears to be slight compared with the strong preference for averageness when the effects of symmetry are controlled for. This supports the proposal that averageness is a more important factor for facial attractiveness than symmetry (Rhodes, 2006).

Although here we have emphasized the role of averageness of 2-D shape for female facial attractiveness, it is important to note that averageness is not the only determinant of attractiveness. Indeed, while the "averageness hypothesis" proposed that average faces are optimally attractive (Langlois \& Roggman, 1990), other research has 
demonstrated that many nonaverage facial cues can have positive effects on attractiveness (e.g., DeBruine, Jones, Unger, Little, \& Feinberg, in press; Perrett et al., 1998; Perrett, May, \& Yoshikawa, 1994). For example, female faces with exaggerated feminine characteristics (e.g., large eyes, full lips) are more attractive than those with more average features (Perrett et al., 1998; Perrett et al., 1994). The relative contributions of average and nonaverage facial characteristics to attractiveness remain to be investigated.

Our findings for stronger averageness preferences when both symmetry and averageness are manipulated than when averageness alone is manipulated suggest that symmetry contributes to the attractiveness of average faces. As such, our findings present converging evidence that facial symmetry is a cue to attractiveness. Additionally, as both symmetry (Cárdenas \& Harris, 2006; Gombrich, 1984) and average configurations (Halberstadt \& Rhodes, 2000; Winkielman et al., 2006) are also preferred in nonface stimuli, our findings raise the possibility that symmetry may also contribute to the appeal of average patterns generally.

\section{AUTHOR NOTE}

Correspondence concerning this article should be addressed to B. C. Jones, Face Research Laboratory, School of Psychology, William Guild Building, University of Aberdeen, Aberdeen AB24 2UB, Scotland (e-mail: ben.jones@abdn.ac.uk.).

\section{REFERENCES}

Alley, T. R., \& Cunningham, M. R. (1991). Averaged faces are attractive, but very attractive faces are not average. Psychological Science, 2, 123-125.

Benson, P. J., \& Perrett, D. I. (1991). Synthesizing continuous-tone caricatures. Image \& Vision Computing, 9, 123-129.

Cárdenas, R. A., \& Harris, L. J. (2006). Symmetrical decorations enhance the attractiveness of faces and abstract designs. Evolution \& Human Behavior, 27, 1-18.

DeBruine, L. M. (2005). Trustworthy but not lust-worthy: Contextspecific effects of facial resemblance. Proceedings of the Royal Society of London: Series B, 272, 919-922.

DeBruine, L. M., Jones, B. C., Unger, L., Little, A. C., \& Feinberg, D. R. (in press). Dissociating averageness and attractiveness: Attractive faces are not always average. Journal of Experimental Psychology: Human Perception \& Performance.

EnQuist, M., Ghirlanda, S., LundQvist, D., \& Wachtmeister, C. A. (2002). An ethological theory of attractiveness. In G. Rhodes \& L. A. Zebrowitz (Eds.), Facial attractiveness: Evolutionary, cognitive and social perspectives (pp. 27-153). Westport, CT: Ablex.

Gombrich, E. H. (1984). The sense of order: A study in the psychology of decorative art. London: Phaidon.

Halberstadt, J., \& RHOdEs, G. (2000). The attractiveness of non-face averages: Implications for an evolutionary explanation of the attractiveness of average faces. Psychological Science, 11, 285-289.

Jones, B. C., DeBruine, L. M., Little, A. C., Conway, C. A., \& FeinBERG, D. R. (2006). Integrating gaze direction and expression in preferences for attractive faces. Psychological Science, 17, 588-591.

Jones, B. C., Little, A. C., Feinberg, D. R., Tiddeman, B. P., PentonVoAK, I. S., \& Perrett, D. I. (2004). The relationship between shape, symmetry, and visible skin condition in male facial attractiveness. Evolution \& Human Behavior, 25, 24-30.

Kampe, K. K., Frith, C. D., Dolan, R. J., \& Frith, U. (2001). Reward value of attractiveness and gaze. Nature, 413, 589.

Langlois, J. H., \& Roggman, L. A. (1990). Attractive faces are only average. Psychological Science, 1, 115-121.

Little, A. C., \& Hancock, P. J. B. (2002). The role of masculinity and distinctiveness in judgements of human male facial attractiveness. British Journal of Psychology, 93, 451-464.

Little, A. C., \& Jones, B. C. (2003). Evidence against perceptual bias views for symmetry preferences in human faces. Proceedings of the Royal Society of London: Series B, 270, 1759-1763.

Maloney, L. T., \& Dal Martello, M. F. (2006). Kin recognition and the perceived facial similarity of children. Journal of Vision, 6, 10471056.

O’Doherty, J., Winston, J., Critchley, H., Perrett, D. [I.], Burt, D. M., \& Dolan, R. J. (2003). Beauty in a smile: The role of medial orbitofrontal cortex in facial attractiveness. Neuropsychologia, 41, 147-155.

O’Toole, A. J., Price, T., Vetter, T., Bartlett, J. C., \& Blanz, V. (1999). 3D shape and 2D surface textures of human faces: The role of "averages" in attractiveness and age. Image \& Vision Computing, 18, 9-19.

Penton-Voak, I. S., \& Perrett, D. I. (2001). Male facial attractiveness: Perceived personality and shifting female preferences for male traits across the menstrual cycle. Advances in Animal Behaviour, 30, 219-259

Perrett, D. I., Burt, D. M., Penton-Voak, I. S., Lee, K. J., Rowland, D. A., \& EDWARDS, R. (1999). Symmetry and human facial attractiveness. Evolution \& Human Behavior, 20, 295-307.

Perrett, D. I., Lee, K. J., Penton-Voak, I. S., Rowland, D. R., YoShikawa, S., Burt, D. M., ET AL. (1998). Effects of sexual dimorphism on facial attractiveness. Nature, 394, 884-887.

Perrett, D. I., May, K. A., \& Yoshikawa, S. (1994). Facial shape and judgments of female attractiveness. Nature, 368, 239-242.

Perrett, D. I., Penton-Voak, I. S., Little, A. C., Tiddeman, B. P., Burt, D. M., Schmidt, N., ET AL. (2002). Facial attractiveness judgements reflect learning of parental age characteristics. Proceedings of the Royal Society of London: Series B, 269, 873-880.

Rhodes, G. (2006). The evolution of facial attractiveness. Annual Review of Psychology, 57, 199-226.

Rhodes, G., Proffitt, F., Grady, J. M., \& Sumich, A. (1998). Facial symmetry and the perception of beauty. Psychonomic Bulletin \& Review, 5, 659-669.

Rhodes, G., Sumich, A., \& Byatt, G. (1999). Are average facial configurations attractive only because of their symmetry? Psychological Science, 10, 52-58.

Rhodes, G., \& Tremewan, T. (1996). Averageness, exaggeration and facial attractiveness. Psychological Science, 7, 105-110.

Rhodes, G., Yoshikawa, S., Clark, A., Lee, K., McKay, R., \& AKaMATSU, S. (2001). Attractiveness of facial averageness and symmetry in nonwestern cultures: In search of biologically based standards of beauty. Perception, 30, 611-625.

Rhodes, G., Zebrowitz, L., Clark, A., Kalick, S. M., Hightower, A., \& MCKAY, R. (2001). Do facial averageness and symmetry signal health? Evolution \& Human Behavior, 22, 31-46.

Rowland, D. A., \& Perrett, D. I. (1995). Manipulating facial appearance through shape and colour. IEEE Computer Graphics \& Applications, 15, 70-76.

Swaddle, J. P., \& Cuthill, I. C. (1995). Asymmetry and human facial attractiveness: Symmetry may not always be beautiful. Proceedings of the Royal Society of London: Series B, 261, 111-116.

Thornhill, R., \& Gangestad, S. W. (1993). Human facial beauty: Averageness, symmetry, and parasite resistance. Human Nature, 4, 237-269.

Tiddeman, B. P., Burt, D. M., \& Perrett, D. I. (2001). Prototyping and transforming facial textures for perception research. IEEE Computer Graphics \& Applications, 21, 42-50.

VAlentine, T., DARling, S., \& Donnelly, M. (2004). Why are average faces attractive? The effect of view and averageness on the attractiveness of female faces. Psychonomic Bulletin \& Review, 11, 482-487.

Winkielman, P., Halberstadt, J., Fazendeiro, T., \& Catty, S. (2006). Prototypes are attractive because they are easy on the mind. Psychological Science, 17, 799-807.

(Manuscript received November 7, 2006; revision accepted for publication April 30, 2007.) 